\title{
Treat to Target in Spondyloarthritis: Myth or Reality?
}

\author{
Authors: \\ *Nelly Ziade, ${ }^{1,2}$ Ihsane Hamamouchi ${ }^{3}$ \\ 1. Saint-Joseph University, Beirut, Lebanon \\ 2. Hotel-Dieu de France Hospital, Beirut, Lebanon \\ 3. Laboratory of Biostatistics, Clinical Research and Epidemiology (LBRCE), Faculty \\ of Medicine and Pharmacy, Mohammed V University, Rabat, Morocco \\ 4. Rheumatology Unit, Provincial Hospital Center, Temara, Morocco \\ *Correspondence to nellziade@yahoo.fr
}

Disclosure: $\quad$ The authors have declared no conflicts of interest.

Received:

Accepted:

Keywords:

Citation:
22.03.21

26.05.21

Axial spondyloarthritis (axSpA), psoriatic arthritis (PsA), spondyloarthritis, treat-totarget, treatment strategy.

EMJ Rheumatol. 2021;8[1]:115-128.

\section{Abstract}

A treat-to-target (T2T) strategy is a treatment plan in which the clinician treats the patient aggressively enough to reach and maintain explicitly specified and sequentially measured goals. To apply a T2T strategy, some conditions should be met. First, a proactive, clear endpoint should be used and a threshold should be defined. Second, a choice between several effective therapies must be available. Third, the endpoint should be supported by findings from randomised controlled trials supporting early aggressive treatment. Fourth, the strategy should be cost-effective. Finally, it needs to be acceptable by the stakeholders.

The objective of this review was to verify if the conditions for applying the T2T strategy were met in psoriatic arthritis (PsA) and axial spondyloarthritis (axSpA), using a narrative review.

Based on the currently available literature, the conditions for applying the T2T in PsA and axSpA were partially met. First, proactive outcome measures are available; however, there is no clear consensus regarding the optimal one. Second, there is a reasonable choice of approved therapies for both diseases. Third, additional randomised controlled trials demonstrating the effectiveness of a T2T approach are still needed. Fourth, cost-effectiveness studies are needed and should include patients from different healthcare systems. Fifth, the implementation of T2T recommendations in routine care and the adherence to its application in clinical practice should be promoted.

In summary, preliminary data suggest that T2T might be beneficial to patients with PSA and axSpA. However, further studies are needed to meet all the criteria before strongly advocating for T2T strategies.

\section{INTRODUCTION}

A treat-to-target (T2T) strategy is a treatment plan in which the clinician treats the patient

aggressively enough to reach and maintain explicitly specified and sequentially measured goals, such as remission, low disease activity, or absence of disability. ${ }^{1,2}$ 
The T2T concept was broadly used in non-rheumatological diseases, such as hypertension, ${ }^{3}$ diabetes, ${ }^{4}$ and dyslipidaemia ${ }^{5}$ and has shown to improve important clinical outcomes such as preventing cardiovascular events, diabetic retinopathy, and even ultimate outcomes such as mortality.

In rheumatology, T2T was applied successfully in rheumatoid arthritis, ${ }^{6}$ in gout, ${ }^{7}$ and in systemic lupus erythematosus, ${ }^{8}$ with clear target cut-offs in disease activity scores and serum urate levels, respectively, correlating with the prevention of radiographic damage. ${ }^{9,10}$

Specific recommendations for T2T in rheumatology were first developed in rheumatoid arthritis in 2010, ${ }^{6}$ followed by recommendations for spondyloarthritis (SpA; including ankylosing spondylitis [AS] and psoriatic arthritis [PsA]) in 2012, which were later updated in 2017." They were adopted, although conditionally, in the international management recommendations for $\mathrm{PSA}^{12,13}$ and axial spondyloarthritis (axSpA). ${ }^{14,15}$ In addition to the goal of optimising the quality of life by decreasing symptoms, inflammation, and structural damage, the T2T recommendations in SpA must face an additional challenge: they must address extra-musculoskeletal manifestations (EMMs) as possible targets, which makes the 'target' a much more heterogeneous and complicated one.
To apply a T2T strategy, some general conditions should be met (Table 1). First, a proactive, clear endpoint, which is the treatment aim, should be used in a specific target algorithm and a threshold should be defined. Second, a choice between several effective therapies that allow the clinical goal to be achieved must be available. Third, the endpoint should be supported by findings from randomised controlled trials (RCTs) suggesting that early aggressive treatment approaches are advantageous. Fourth, the proposed strategy should be cost-effective. Fifth, it needs to be acceptable by the stakeholders. ${ }^{16}$

Although the T2T approach is well established in RA, its relevance and applicability in PsA and axSpA are still debated.

\section{OBJECTIVE}

The objective of this review was to verify if the conditions for applying the T2T strategy were met in PsA and in axSpA.

\section{METHODS}

Using the key words "treat to target", "rheumatology", and "spondyloarthritis" in PubMed, the authors conducted this narrative review. First, the authors identified the conditions that are required to apply a T2T strategy and summarised them in five questions.

Table 1: Summary of conditions needed to apply a T2T strategy in psoriatic arthritis and axial spondyloarthritis.

\begin{tabular}{|c|c|c|}
\hline Conditions for T2T & Psoriatic arthritis & Axial spondyloarthritis \\
\hline $\begin{array}{l}\text { Is there a proactive, clear endpoint, } \\
\text { which is the aim of the treatment? }\end{array}$ & $\begin{array}{l}+ \\
\text { (A consensus regarding the best } \\
\text { target is needed) }\end{array}$ & $\begin{array}{l}+ \\
\text { (A consensus regarding the best } \\
\text { target is needed) }\end{array}$ \\
\hline $\begin{array}{l}\text { Is there a choice of several effective, } \\
\text { available therapies that allow the } \\
\text { clinical target to be reached? }\end{array}$ & + & + \\
\hline $\begin{array}{l}\text { Is the endpoint supported by findings } \\
\text { from RCTs suggesting that early, } \\
\text { aggressive treatment approaches } \\
\text { would be advantageous? }\end{array}$ & $\begin{array}{l}+/- \\
\text { (One RCT, soft endpoints) }\end{array}$ & $\begin{array}{l}+/- \\
\text { (One RCT, soft endpoints) }\end{array}$ \\
\hline Is the strategy cost-effective? & (One RCT) & $\begin{array}{l}+ \\
\text { (One RCT) }\end{array}$ \\
\hline $\begin{array}{l}\text { Is the strategy acceptable by the } \\
\text { stakeholders? }\end{array}$ & $+/-$ & $+/-$ \\
\hline
\end{tabular}

RCT: randomised controlled trial; T2T: treat-to-target. 
Then, the responses to these five questions were sought for PsA and axSPA, respectively.

Is there a proactive, clear endpoint, which is the aim of the treatment?

A 'good' endpoint or target must be easily measurable in clinical practice, be validated in patients with PsA and axSpA, respectively, and reflect clinical outcomes that are important to both patients and physicians. The choice of the target should be a shared decision between the patient and the rheumatologist, considering all relevant situational factors. Treatment, once started, should be monitored to investigate if the endpoint is reached. The endpoint should be used in a specific target algorithm, and a threshold should be defined. Since both diseases, particularly PsA, are very heterogeneous, encompassing arthritis, enthesitis, dactylitis, and/or psoriasis in the same patient, finding the optimal target is challenging.

The targets can be soft, reversible outcomes (i.e., disease activity score or inflammatory markers) or hard, irreversible outcomes (i.e., radiographic damage or disability). ${ }^{11}$ In most cases, soft endpoints correlate with the hard outcomes while being easier to obtain, thus often serving as surrogate measures for the hard, more relevant outcomes. ${ }^{17-21}$

Moreover, the clinician must keep in mind the EMMs and take them into account when facing a specific clinical situation.

\section{Is there a choice of several effective, available therapies that allow the clinical target to be reached?}

A treatment is usually considered effective when its value has been demonstrated by high-quality RCTs. For the current analysis, the authors included the therapies that are recommended by international rheumatology bodies such as the American College of Rheumatology $(\mathrm{ACR}) /$ National Psoriasis Foundation (NPF), ${ }^{13}$ the European Alliance of Associations for Rheumatology (EULAR), ${ }^{12,14}$ the Assessment of Spondyloarthritis International Association (ASAS), ${ }^{14}$ and the Group for Research and Assessment of Psoriasis and Psoriatic Arthritis (GRAPPA). ${ }^{22}$
Any therapy whose efficacy was demonstrated in a recent RCT published after issuing these recommendations was also evaluated. Nonpharmacological treatments were evaluated, as well as a mean to treatment optimisation.

\section{Is the endpoint supported by findings from RCTs suggesting that early aggressive treatment approaches would be advantageous?}

RCTs comparing the T2T strategy to the standard of care in PsA and axSpA, respectively, were reviewed.

\section{Is the strategy cost-effective?}

Cost-effectiveness studies were analysed from the identified RCTs.

\section{Is the strategy acceptable by the stakeholders?}

Acceptability was first evaluated by checking if the T2T strategy was adopted in the PsA and the axSpA recommendations (ACR, ASAS, GRAPPA, and EULAR). Second, studies regarding the implementation of T2T in clinical practice and its related perceptions by patients and by healthcare providers (HCP) were analysed.

Finally, strategies for implementing and adopting T2T in clinical practice were discussed, and the unmet needs and areas for future research were identified.

\section{RESULTS}

\section{Psoriatic Arthritis}

Is there a proactive, clear endpoint, which is the aim of the treatment?

Many composite measures exist and can be potential candidates for use as a proactive endpoint.11,23 These measures include ACR outcome measure, Arithmetic mean Desirability Function (AMDF) composite score, Composite Psoriatic Disease Activity Index (CPDAI), Disease Activity in Psoriatic Arthritis (DAPSA), Disease Activity Score 28 joints (DAS-28:), Group for Research and Assessment of Psoriasis and Psoriatic Arthritis Composite Index (GRACE), Minimal Disease Activity (MDA), Psoriatic Arthritis Disease Activity Score 
(PASDAS), Psoriatic Arthritis Response Criteria (PsARC), Routine Assessment of Patient Index Data 3 (RAPID3), and Very Low Disease Activity (VLDA) (Table 2). All of these measures include joint counts: tender (TJC) and swollen (SJC). Many scores include the patient's and/or the physician's global assessment; some of them include inflammatory markers, skin outcomes, or evaluation of dactylitis, enthesitis, or axial involvement.

Overall, according to two systematic literature reviews, there is an important heterogeneity regarding the composite outcome measure used in PsA studies; therefore, a consensus in this area is a clear unmet need. ${ }^{24,25}$ In a GRAPPA meeting including 26 rheumatologists, dermatologists, and patient research partners, the panel could not reach a consensus regarding a continuous measure of disease activity. ${ }^{23}$

A comparison of remission and low disease activity states with DAPSA, MDA, and VLDA in a clinical trial setting in patients with PsA concluded that both DAPSA and MDA composite measures (Table 3 ) can be used for evaluation of the status and treatment response utilising a T2T approach and can improve patient healthrelated outcomes. These two measures were also the ones that were preferred in the 2017 T2T recommendations." Likewise, in a clinical trial setting of the study using DAPSA and MDA in secukinumab-treated patients with PsA, both composite measures were useful for evaluation of the status and treatment response utilising a T2T approach. ${ }^{26}$

On the one hand, the DAPSA was initially developed for reactive arthritis. ${ }^{27}$ It has been validated for use in PsA, where it showed correlational, discriminatory, and criterion validity; furthermore, it was sensitive to change in trials and observational studies alike and has shown a good correlation with ultrasoundassessed synovitis. ${ }^{28,29}$ Schoels et al. ${ }^{30}$ provided criteria for disease activity states and treatment response, which showed good performance in clinical trials and observational data. It is a simple measure and specifically measures peripheral arthritis without the inclusion of any other domains. Therefore, a separate assessment of skin disease and potentially other domains should be mandated alongside the DAPSA score to ensure a full assessment of PsA disease activity. DAPSA use was recommended by the T2T $2017^{11}$ and the ACR $2018^{13}$ PsA management recommendations.

On the other hand, the MDA encompasses several disease domains (joint counts, global assessment, skin assessment, $\mathrm{HAQ}$, enthesitis) and is increasingly accepted. The MDA criteria were specifically developed with the idea of investigating the benefits of T2T in $\mathrm{PsA}^{31}$ and were validated in PsA in 2010 and used as a key outcome measure in the main T2T trial in PsA. ${ }^{32}$ MDA use was recommended by the GRAPPA 2015, ${ }^{22}$ the T2T 2017,11 and the ACR 2018 ${ }^{12}$ management recommendations. In a recent analysis, Mease et al. ${ }^{33}$ compared the disease control thresholds in the Corrona PsA/axSpA registry. They confirmed the previously described notion that MDA and VLDA were the most stringent disease activity measures and resulted in overall lower disease activity in multiple key domains compared to patients who met clinical DAPSA, Patient Acceptable Symptom State (PASS), Patient Global Assessment of Arthritis (PtGA), and Patient Global Assessment of Arthritis and Psoriasis (PtGA PA) thresholds. ${ }^{25}$ Therefore, they encouraged the rheumatologists to use MDA/VLDA to assess disease control in patients with PsA.

Furthermore, since PsA is a very heterogeneous disease, recent head-to-head trials have used combined outcomes to reflect the complexity of the disease, including targets in the joint $(A C R)$ and the skin measurements (Psoriasis Area and Severity Index; PASI) that must be reached simultaneously. ${ }^{34,35}$ This approach has helped distinguish some patients' profiles where a specific therapeutic class can be effective. Moreover, patients simultaneously achieving ACR 50\% improvement (ACR50) and PASI 100\% improvement (PASI100) had consistently better improvements in other T2T outcomes, including MDA and VLDA. ${ }^{36}$

All the mentioned scores are only surrogates for the hard endpoints, which mainly include radiographic damage and long-term disability and represent the ultimate goal of any therapy in PsA. ${ }^{11}$ However, these hard endpoints are slow to achieve and their inclusion in a T2T strategy, where timely interventions are needed, remains challenging. 


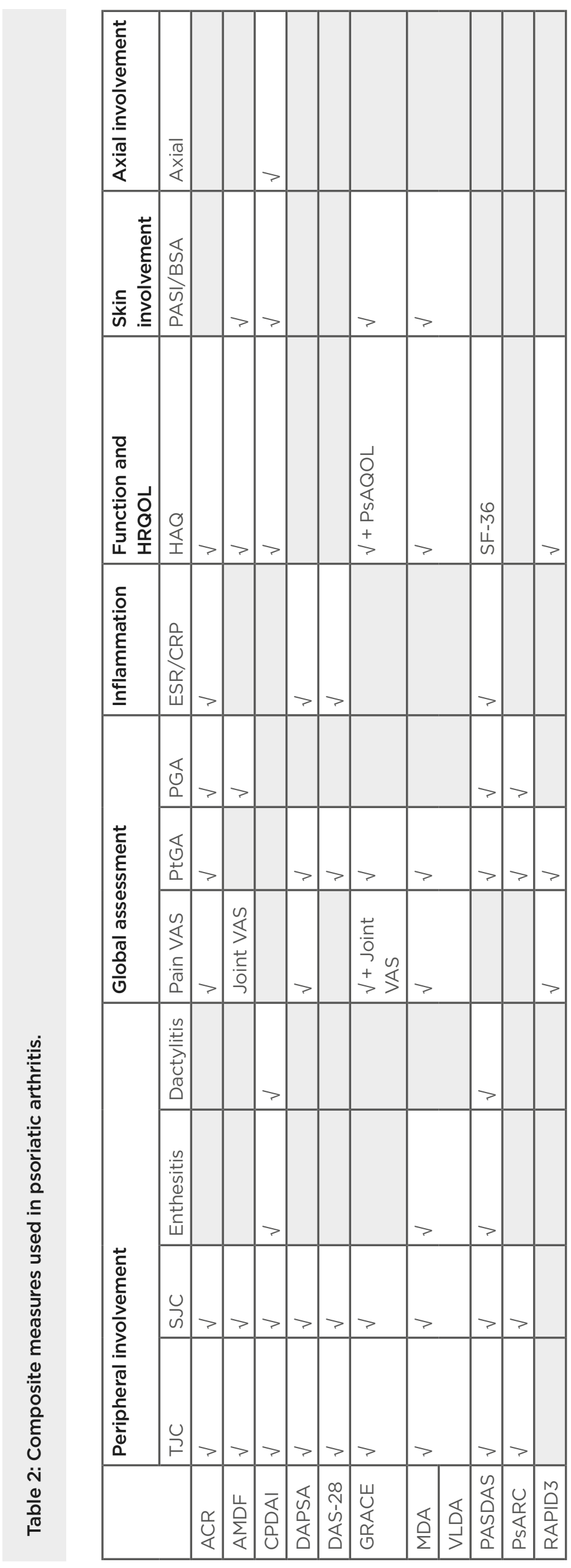

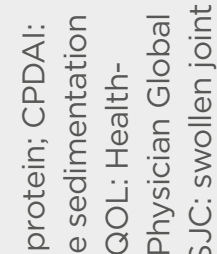

웡

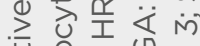

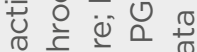

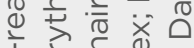

u过 ᄃ

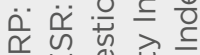

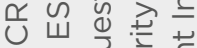

i in 0 वे ब

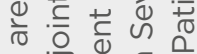

ช)

议 $N$

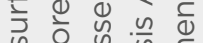

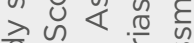

ठำ

$\Omega$

远宬空㐫

$\infty \varangle \ddot{0} \varangle$

बi

이워

ज市 讪

$\stackrel{4}{\dot{n}} \dot{\infty} \subseteq \underset{0}{2}$

ก $N \geq \frac{1}{2}$

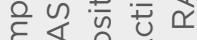

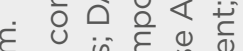

है ज है

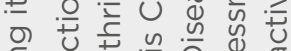

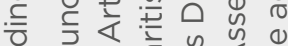

ᄃ

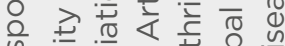

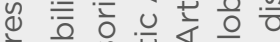

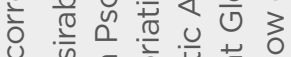

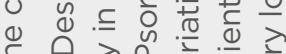

म

吕

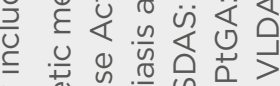

○ ह

ज 듬

잉

1)

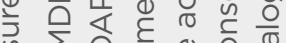

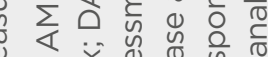

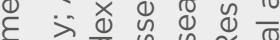

Q $0 \leq \varangle \frac{1}{0}$ ज

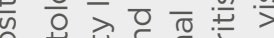

官

ह ह

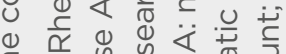

1)

+ 0 o

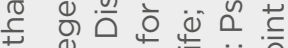

() $\overline{\overline{0}}$ 을

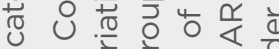

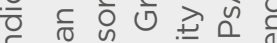

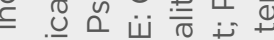

ص ब ब

$x$ ह ज्ञ त ह ए

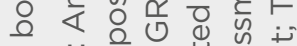

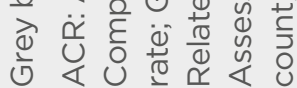


Table 3: MDA criteria and DAPSA are the most recommended outcome measures in psoriatic arthritis.

\begin{tabular}{|c|c|}
\hline Outcome measure in PsA & Calculation \\
\hline \multicolumn{2}{|l|}{ MDA } \\
\hline $\begin{array}{l}\text { Tender joint count } \leq 1 \text { (out of } 68 \text { assessed) } \\
\text { Swollen joint count } \leq 1 \text { (out of } 66 \text { assessed) } \\
\text { PASI } \leq 1 \text { or BSA } \leq 3 \% \\
\text { Patient's assessment of pain (VAS) } \leq 15 \\
\text { Patients' global assessment of disease activity (VAS) } \leq 20 \\
\text { HAQ-DI } \leq 0.5 \\
\text { Tender entheseal points } \leq 1\end{array}$ & $\begin{array}{l}\text { A patient is classified as in MDA when they meet } 5 \text { of the } 7 \\
\text { criteria. } \\
\text { A patient is classified in VLDA when they meet all } 7 \text { criteria. }\end{array}$ \\
\hline \multicolumn{2}{|l|}{ DAPSA } \\
\hline $\begin{array}{l}\text { Tender joint count (out of } 68 \text { assessed) } \\
\text { Swollen joint count (out of } 66 \text { assessed) } \\
\text { CRP (mf/dL) } \\
\text { Patient's assessment of disease activity (NS O-10) } \\
\text { Patient's assessment of pain (NS 0-10) }\end{array}$ & $\begin{array}{l}\text { DAPSA score is the sum of all the above. } \\
\text { O- } 4 \text { remission, } 5-14 \text { low, } 15-28 \text { moderate, }>28 \text { high disease } \\
\text { activity. }\end{array}$ \\
\hline
\end{tabular}

BSA: body surface area; DAPSA: Disease Activity in Psoriatic Arthritis; HAQ-DI: Health Assessment Questionnaire Disability Index; MDA: minimal disease activity; NS: numerical scale; PASI: Psoriasis Area Severity Index; VAS: visual analogue scale; VLDA: very low disease activity.

Beyond the discussed endpoints, EMMs are also essential to address. The prevalence of common EMMs in PsA is 90\% for psoriasis, $3-7 \%$ for inflammatory bowel diseases, and 1-3\% for uveitis. ${ }^{37,38}$ They should be identified and managed in a multidisciplinary setting as their presence may significantly impact the treatment decision.

Finally, the choice of the target of the disease activity should take comorbidities, patient factors, and drug-related risks into account."

\section{Is there a choice of several effective available therapies that allow the clinical target to be reached?}

The choice of therapies for PsA has tremendously increased during the last decade. Several effective therapies are now approved, and many others are under study (Table 4).

According to the EULAR, ACR/NPF, and GRAPPA recommendations, ${ }^{12,13,22}$ conventional synthetic disease-modifying drugs (cs-DMARDs, usually methotrexate) can be used after the failure of non-steroidal anti-inflammatory drugs
(NSAIDs), except when axial disease and/or enthesitis are predominant. After the failure of cs-DMARDs, the biologic therapies (antiTNFa, anti-IL-12/IL-23, anti-IL-17A) and the small molecules (JAK inhibitors, phosphodiesterase type 4 inhibitors) are recommended. They should be prioritised according to the main domain involved and several treatment algorithms were proposed by the international, regional, and local recommendations. More recent data regarding anti-IL23-p19 seem to be very promising, expanding the therapeutic armamentarium furthermore. ${ }^{39-41}$

\section{Is the endpoint supported by findings from RCTs suggesting that early aggressive treatment approaches would be advantageous?}

Evidence of the effectiveness of the T2T strategy was assessed in PsA in a key trial, the TICOPA study, ${ }^{32}$ published in 2015.

TICOPA was an open-label study conducted in the UK and included 206 patients who were DMARD-naïve and had a PsA of short duration (<2 years). The study duration was 48 weeks. 
Table 4: Effective therapies in psoriatic arthritis, axial spondyloarthritis, and extra-musculoskeletal manifestations.

\begin{tabular}{|c|c|c|c|c|c|c|}
\hline & $\begin{array}{l}\text { Psoriatic } \\
\text { arthritis }\end{array}$ & axSpA & Psoriasis & $\begin{array}{l}\text { Crohn's } \\
\text { disease }\end{array}$ & $\begin{array}{l}\text { Ulcerative } \\
\text { rectocolitis }\end{array}$ & Uveitis \\
\hline TNFa inhibitors & ++ & ++ & ++ & $\begin{array}{l}\text { ++ (except } \\
\text { etanercept, } \\
\text { golimumab) }\end{array}$ & $\begin{array}{l}\text { ++ (except } \\
\text { etanercept, } \\
\text { certolizumab) }\end{array}$ & $\begin{array}{l}\text { ++ } \\
\text { (adalimumab, } \\
\text { infliximab) }\end{array}$ \\
\hline $\begin{array}{l}\text { IL-17A } \\
\text { inhibitors }\end{array}$ & ++ & $\begin{array}{l}\text { ++ } \\
\text { (secukinumab, } \\
\text { ixekizumab) }\end{array}$ & ++ & -- & -- & $?$ \\
\hline $\begin{array}{l}\text { IL-12/ } \\
\text { IL-23 inhibitors }\end{array}$ & ++ & - & ++ & ++ & + & $?$ \\
\hline IL23 inhibitors & + & - & ++ & + & + & $?$ \\
\hline JAK inhibitors & ++ & + & + & + & ++ & $?$ \\
\hline PDE4 inhibitors & ++ & - & ++ & ? & + & ? \\
\hline
\end{tabular}

\begin{tabular}{|l|l|}
\hline++ & FDA-approved \\
\hline+ & Peliminary data on clinical efficacy \\
\hline$?$ & Not studied/insufficient data \\
\hline- & Failed to meet primary endpoint \\
\hline-- & Disease-aggravating effect \\
\hline
\end{tabular}

axSpA: axial spondyloarthritis; FDA: U.S. Food and Drug Administration; PDE4: phosphodiesterase 4.

The patients were randomised to either standard therapy with 3-monthly evaluations with no strict guidance about the treatment decisions, or tight control (TC) with 4-weekly evaluations and step-up therapy (starting with methotrexate and stepping-up to adalimumab) if MDA was not reached. At Week 12, MDA was achieved in $24 \%$ of the TC group. The proportion of patients reaching ACR20, ACR50, ACR70, and PASI75 was significantly higher in the TC group. Moreover, a significantly greater improvement was observed for patient-reported outcomes in the TC arm. Regarding the treatments, the use of biologics was much higher in the TC group, and this group had a much higher incidence of adverse events.

Regarding radiographic progression, although it was numerically lower in the TC group, it did not reach statistical significance at Week 48 . It was argued that the included population had mild disease with low baseline radiographic scores and consequently a low risk for radiographic progression. ${ }^{42}$
Other studies where therapy was altered based on achieving a target were conducted with anti-TNF clinical trials. ${ }^{24}$ They included a plan in the study protocol for an escalation of treatment if pre-specified targets were not met, labeled as an 'early escape' arm where patients at a set time point (12 or 16 weeks), still in the double-blind portion of the study, could be rerandomised to potentially increase therapy if a particular reduction in their disease activity was not met. The target used in these studies was the reduction in the number of tender and swollen joints, which is a questionable endpoint. Moreover, these studies were not investigating the impact of $\mathrm{T} 2 \mathrm{~T}$ in a robust comparison against standard care.

Therefore, there is a clear need for additional RCTs that investigate the value of T2T strategies in PsA. Moreover, evidence on the effect of these strategies on the long-term outcomes, namely radiographic damage, function, and healthrelated quality of life, is essential. 


\section{Is the strategy cost-effective?}

An analysis from the TICOPA study ${ }^{32}$ from the perspective of the UK National Health System (NHS) found that when this strategy was applied in a nation-wide sample, the incremental cost-effective ratio was 54,000 GBP $(70,200$ USD) per quality-adjusted life year (QALY), which exceeded the threshold allowable by the NHS and drove the authors to conclude that T2T strategy in PsA was not cost-effective. ${ }^{43}$ The analysis did not incorporate indirect costs to patients, such as productivity loss; incorporating such costs likely would make tight control even less favourable due to its expense.

\section{Is the T2T strategy acceptable by the stakeholders?}

The T2T concept was adopted by the 2018 GRAPPA recommendations for the use of composite measures and treatment targets in PSA, ${ }^{23}$ the ACR 2018,13 and the EULAR 2020 PsA management recommendations. ${ }^{12}$ The ACR gave only a conditional recommendation for the use of the T2T strategy over not following a T2T strategy, and stated that one might consider not using a T2T strategy in patients in whom there are concerns related to increased adverse events, costs of therapy, and patient burden of medications associated with tighter control. The latest EULAR recommendations updated in 2020 rephrased their T2T recommendation. They specified that the target should be remission or low disease activity (instead of minimal disease activity), while acknowledging the difficulty of defining remission and suggesting using the abrogation of inflammation as an indicator of remission. They gave this recommendation a Grade A, with a high level of agreement (9.4).

When considering whether T2T is applied in practice, studies showed that it was adopted by only a minority of patients. ${ }^{44}$ From the patients' perspective, they may have adapted to their disease and became reluctant to change if they feel 'OK' even if they still have some disease activity. Also, they might disagree with the physician's measure of their disease activity. Furthermore, some patients who are required to make out-of-pocket contributions to healthcare might be unwilling to visit their rheumatologist more frequently.
Regarding the HCPs, a GRAPPA survey showed that $56 \%$ reported that they do T2T in clinical practice. ${ }^{23}$ Also, a qualitative study of clinicians' perspectives identified the barriers to implementation of T2T in PsA using interviews with rheumatologists and other healthcare professionals: ${ }^{45}$ individual motivation to change clinical practice, lack of consensus on what to measure, what is achievable with limited resources, and mandatory versus voluntary pressures to change. Moreover, T2T requires frequent visits and the use of standardised outcomes measures, which may be challenging for rheumatologists with busy practices.

\section{Axial Spondyloarthritis}

\section{Is there a proactive, clear endpoint, which is the aim of the treatment?}

For the first time in the history of SpA research, evidence has been accrued to suggest the value of 'targeting disease activity' because disease activity leads to new syndesmophytes in patients with axSpA. ${ }^{17,18}$

Many endpoints were proposed in T2T strategies in axSpA: from markers of disease activity (C-reactive protein [CRP], Bath Ankylosing Spondylitis Activity Index [BASDAI], ${ }^{46}$ Ankylosing Spondylitis Disease Activity Score [ASDAS], ${ }^{47-49}$ Assessment of Spondyloarthritis International Association [ASAS] Remission); 50 to markers of structural progression, disability (ASAS Health Index; ASAS-HI), ${ }^{51}$ as well as comorbidities (smoking cessation, NSAID intake, hypertension, diabetes).

Also, these include EMM and sequelae of the long-standing disease, such as cardiovascular disease and osteoporosis.

The two most-used target measures for axSpA in clinical practice are the BASDAI and the ASDAS (Table 5). ${ }^{52}$

The ASDAS was developed to attempt to overcome some of the limitations of the BASDAI. Indeed, due to the subjectivity of the items included on the BASDAl, there is often discordance between patient and clinician assessments of the disease activity. ${ }^{53}$ ASDAS includes some questions from the BASDAI as well as patient and physical global assessments and laboratory measures (either the CRP or the erythrocyte sedimentation rate [ESR]). 
Table 5: Bath Ankylosing Spondylitis Activity Index (BASDAI) and Ankylosing Spondylitis Disease Activity Score (ASDAS) are the most recommended outcome measures in axial spondyloarthritis.

\section{Outcome measure in axSpA \\ BASDAI \\ 1. How would you describe the overall level of fatigue/ tiredness you have \\ experienced? \\ 2. How would you describe the overall level of ankylosing spondylitis neck, \\ Back, or hip pain you have had? \\ 3. How would you describe the overall level of pain/swelling in joints other \\ than neck, back, or hips you have had? \\ 4. How would you describe the overall level of discomfort you have had from \\ any areas tender to touch or pressure? \\ 5. How would you describe the overall level of morning \\ stiffness you have had \\ from the time you wake up? \\ 6 . How long does your morning stiffness last from the time you wake up?}

ASDAS inactive disease

1. How would you describe the overall level of AS neck,

back, or

hip pain you have had?

2. How active was your spondylitis on average?

3. How would you describe the overall level of pain/swelling in joints other than neck, back, or hips you have had?

4. How long does your morning stiffness last from the time you wake up?

5. CRP measured in $\mathrm{mg} / \mathrm{L}$ or ESR

\section{Calculation}

Assess each question on a NRS of $O$ (none) to 10 (very severe); alternatively, a VAS can be used for questions 1-5 (NRS preferred by ASAS)

Calculation of BASDAl:

- Compute the mean of questions 5 and 6.

- Calculate the sum of the values of questions 1-4 and add the result to the mean of questions 5 and 6 .

- Divide the result by 5 .

A BASDAI score $\geq 4 / 10$ is considered as the threshold above which a disease status can be considered as 'active'. A change of at least $50 \%$ in the BASDAI is usually considered as reflecting a clinically relevant improvement

ASDAS-CRP $=0.121 \times$ total back pain $+0.110 \times$ patient global $+0.073 \times$ peripheral pain/swelling $+0.058 \times$ duration of morning stiffness $+0.579 \times \ln (\mathrm{CRP}+1)$;

ASDAS-ESR $=0.113 \times$ patient global $+0.293 \times \sqrt{ }$ ESR +0.086 $x$ peripheral pain/swelling $+0.069 x$ duration of morning stiffness $+0.079 \times$ total back pain

Assess each question on an NRS of O (none) to 10 (very severe)

ASDAS inactive disease is $<1.3$

ASAS partial remission

A value not above two units on a 0-10 scale in four of four ASAS domains

1. Physical function (BASFI)

2. Pain (by VAS)

3. Inflammation (morning stiffness)

4. Patient global assessment (by VAS)

ASAS: Assessments of Spondyloarthritis International Society; ASDAS: Ankylosing Spondylitis Disease Activity Score; BASDAI: Bath Ankylosing Spondylitis Disease Activity Index; BASFI: Bath Ankylosing Spondylitis Functional Index; CRP: C-reactive protein; ESR: erythrocyte sedimentation rate; NRS: numerical rating scale; VAS: visual analogue scale.

However, the ASDAS has some limitations: it does not incorporate other objective measures of inflammation, such as those found on imaging. ASDAS has validated thresholds for disease activity categories, whereas BASDAI does not, and it is the preferred measure in axSpA according to T2T international task force recommendations. ${ }^{11}$ 
Recent studies showed that achievement of an inactive disease status (ASDAS 1.3) while on treatment with anti-TNFa resulted in almost complete radiographic spinal progression inhibition during the following 2-year radiographic interval.16,19 Other anti-TNFa trials showed that $15-35 \%$ of patients reach ASDASInactive Disease (ASAS-ID). ${ }^{20,21}$ ASAS partial remission can be used, but its main limitation is that it relies partly on the Bath Ankylosing Spondylitis Functional Index (BASFI), and a patient with irreversible structural damage may be unable to fulfill ASAS partial remission criteria. ${ }^{54}$ ASDAS low disease activity can also be a therapeutic target, ${ }^{11,16}$ but it is still debated because of the lack of data. ${ }^{52}$

In response to the lack of a definition of AS disease severity, ASAS developed an instrument based on the International Classification of Functioning, Disability, and Health (ICF) model of function and health, the ASAS-HI. ${ }^{51} \mathrm{~A}$ value $\geq 12$. 0 serves as the cut-off between poor and moderate health, whereas a value $<5.0$ is the cut-off between good and moderate health. The ASAS-HI serves as the primary outcome measure in a recent $\mathrm{T} 2 \mathrm{~T}$ trial in axSpA. ${ }^{55}$

As for the structural damage evaluation, it could be performed by sacroiliac and spine radiographs and also by MRI. Some have proposed that imaging could be used in patients in clinical remission in whom tapering of biologics is considered. ${ }^{2}$ However, this suggestion was refuted by Smolen et al. ${ }^{11}$ because there are no data justifying the use of imaging in follow-up yet, and it is not feasible to perform $\mathrm{MRI}$ repeatedly in axSpA.

Less-conventional outcomes, such as smoking cessation, NSAID use, and cardiovascular disease, require a long follow-up time and are therefore not easy to assess in RCTs but can be assessed in prospective cohort studies. ${ }^{2}$ However, such information would be relevant to understand the effects of treatment on long-term complications of axSpA and to optimise the T2T approach, especially in cases where access to different biotherapies is not simple.

As mentioned earlier, the choice of the target and of the disease activity should take comorbidities, patient factors, and drug-related risks into account. ${ }^{11}$
As for the EMMs, according to a meta-analysis, the pooled lifetime prevalence of common EMMs in patients with axSpA were $26 \%$ for uveitis, $9 \%$ for psoriasis, and 7\% for inflammatory bowel disease. ${ }^{56}$ They should also be evaluated using a multidisciplinary approach.

\section{Is there a choice of several effective, available therapies that allow the clinical target to be reached?}

As with PsA, the choice of therapies for axSpa has increased considerably over the past decade and several effective therapies are now approved (Table 3).

According to the EULAR-ASAS ${ }^{14}$ and the $A C R^{15}$ recommendations, after NSAIDs failure, the biologic therapies (anti-TNFa, anti-IL-17A) are recommended. They should be prioritised according to the main domain involved, and several treatment algorithms were proposed by the international recommendations. Data from several retrospective observational studies analysis suggested that anti-TNF therapy can delay the radiographic progression in the long term. ${ }^{19,57,58}$ Additionally, tofacitinib, a JAK inhibitor, has shown promising results, adding to the armamentarium of the treatment of axSpA. ${ }^{59}$

\section{Is the endpoint supported by findings from RCTs suggesting that early aggressive treatment approaches would be advantageous?}

To date, there have been two T2T trials in axSpA.

The STRIKE study ${ }^{60}$ was a German RCT of patients with axSpA meeting the ASAS axSpA criteria and having been symptomatic for $<5$ years, who were randomised to T2T versus usual care. In the T2T arm they were assessed monthly, and the protocol involved starting with an NSAID and escalating to adalimumab. The primary outcome was ASDAS inactive disease (ASDASID) at 32 weeks. Unfortunately, this trial was stopped due to slow recruitment. ${ }^{52}$

TICOSPA $^{55}$ is a European pragmatic, prospective, cluster-randomised controlled trial of patients with axSpA, comparing tight control with monthly assessments to usual care for one year. The primary outcome is change in the ASAS-HI over 1 year. Secondary outcome measures include ASDAS, BASDAI, quality of life, and resource 
utilisation. The strategy was pre-specifed by the scientific committee based on current axSpA recommendations and aiming at a target of ASDAS <2.1, with visits every 4 weeks. The treatment decisions in usual care arms were at the rheumatologists' discretion, with visits every 12 weeks. One hundred and sixty patients were included ( 80 in TC and 80 in usual care). The mean age was 37.9 $( \pm 11.0)$ years with a disease duration of $3.7( \pm 6.2)$ years. 51.2\% were males. Radiographic damage of the sacroiliac joints, an (ever) positive MRI sacroiliitis, and HLA-B27+ were seen in 46.9\%, $81.9 \%$, and $75.0 \%$ of patients, respectively. Mean ASDAS at inclusion was $3.0( \pm 0.7)$ and mean ASAS-HI was 8.6 ( \pm 3.7$)$. Although $47.3 \%$ versus $36.1 \%$ patients in the TC and usual care arms achieved an improvement in ASAS-HI at the 1-year visit, which was considered clinically relevant, the difference was not statistically significant. Across all other outcomes, a trend was observed in favor of the TC arm. The number of biological DMARDs was significantly higher in the T2T arm (56.2\% versus $27.2 \%$ ). The number of infections was comparable in both groups (15 versus 16 in the TC and usual care arms, respectively).

\section{Is the strategy cost-effective?}

To date, there is one cost-effectiveness analysis of T2T strategies in axSpA. Indeed, an analysis from the TICOSPA study found that when this strategy was applied, the T2T strategy was cost-effective with an incremental costeffective ratio of 19,430 EUR. From a societal perspective, T2T resulted in an additional 0.04 QALY and saved 265 EUR when compared to usual care and a $67 \%$ probability of being costeffective at a cost-effectiveness threshold of 20,000 EUR per QALY.55

\section{Is the strategy acceptable by the stakeholders?}

Smolen et al. ${ }^{11}$ indicated that with T2T strategies, all the options were acceptable; namely, to be left as they had been initially constructed, amended, deleted, or expanded in number and/ or changed in sequence. But the lack of evidence available led some societies, such as ACR, not to recommended $\mathrm{T} 2 \mathrm{~T}$ strategies in axSpA.

Indeed, the ACR 2019 conditionally recommended the regular-interval use and monitoring of a validated AS disease activity measure, and conditionally recommended regular-interval use and monitoring of CRP concentrations or ESR over usual care without regular CRP or ESR monitoring.

For adults with active AS or non-radiographic axSpA, they conditionally recommended against using a T2T strategy using a target of ASDAS $<1.3$ (or 2.1) over a treatment strategy based on physician assessment. For patients and providers, the panel felt that more convincing evidence of benefit should be present before approving this change in practice. Their rationale was that they feared that choosing a specific target would lead to rapid cycling of all currently available treatments in some patients. That said, they emphasised the importance of having targets in the management of patients. ${ }^{61}$

The EULAR/ASAS 2016 recommendations recommended that a target should be defined and documented, but, unlike the T2T international task force and the ACR guidelines, ${ }^{11,61}$ refrained from mentioning the content of such target. This target may change depending on the phase of the disease and the treatments already used previously.

\section{DISCUSSION}

Based on the currently available literature, the conditions for applying the T2T in PsA and axSpA are partially met.

First, proactive outcome measures are available, however, there is no clear consensus regarding the choice of the optimal measure. Using a universal target allows for better comparability between the clinical trials. Moreover, soft endpoints should be validated against a gold-standard hard endpoint (such as long-term disability, quality of life measures, and/or radiographic damage). DAPSA and MDA in PsA and ASDAS in axSpA should be correlated with radiographic scores and long-term measures of disability to properly estimate the effect of a treatment strategy on the general burden of the disease, respectively. Acceptable cut-off scores for soft outcomes, in relationship to these hard outcomes, should be adopted on larger scales. Furthermore, the inclusion of radiologic measures and 
clinical activity related to EMMs in T2T studies should be discussed but may require longer follow-up studies.

Second, there is a choice of several available therapies for both diseases, and the treatment armamentarium is constantly increasing.

Third, additional RCTs demonstrating the effectiveness of a T2T approach in providing an advantage over the standard care are still needed. Researchers should continue to evaluate whether current therapeutic tools are sufficient to reach the proposed targets and investigate the benefit from the active implementation of non-pharmacological treatments in the T2T strategies.

Fourth, cost-effectiveness studies are needed and should include patients from different healthcare systems. Also, the inclusion of nonpharmacological treatments, particularly in settings of low economic resources, should be considered.

Fifth, the implementation of T2T recommendations in routine care and the adherence to its application in clinical practice should be promoted. ${ }^{62,63}$ Financial constraints, staff shortages, patients' reluctance, and high clinic demands are among the reasons for implementation difficulties. Many methods are available to implement T2T in clinical practice. They rank from the least to the most effective: education, rules and policies, reminders and checklists, simplification and standardisation, and forcing functions. ${ }^{2}$ T2T may also be successfully implemented if rheumatologists are required to enter detailed data into registries. ${ }^{64}$ Moreover, the role of non-physician HCPs such as rheumatology nurses in the implementation of T2T in clinical practice should be evaluated, following very successful experience in diabetes, hypertension, and hyperlipidaemia. ${ }^{65,66}$ Furthermore, the role of electronic health records that prompt rheumatologists about escalation/de-escalation opportunities and capture their medical decision-making could allow the development of refined T2T care strategies and deserves further evaluation. ${ }^{67}$

\section{CONCLUSION}

In summary, T2T is an emerging management strategy in PsA and axSpA. Preliminary data suggest that a T2T approach might be beneficial to patients with PsA and axSpA. However, further studies are needed to meet all the required criteria before strongly advocating for T2T strategies in clinical practice.

\section{References}

1. Solomon $\mathrm{DH}$ et al. Review: treat to target in rheumatoid arthritis: fact, fiction, or hypothesis? Arthritis Rheumatol. 2014;66:775-82.

2. Dougados M. Treat to target in axial spondyloarthritis: from its concept to its implementation. J Autoimmun. 2020;110:102398

3. Whelton PK et al. 2017 ACC/AHA/ AAPA/ABC/ACPM/AGS/APhA/ ASH/ ASPC/NMA/PCNA guideline for the prevention, detection, evaluation, and management of high blood pressure in adults a report of the American College of Cardiology/ American Heart Association Task Force on clinical practice guidelines. Hypertension. 2018;71(6):1269-324.

4. Riddle $\mathrm{M}$ et al. The treat-to-target trial. Diabetes Care. 2003;26:3080-6.

5. Grundy SM et al. 2018 AHA/ACC/ AACVPR/AAPA/ABC/ACPM/ADA/ AGS/APhA/ASPC/NLA/PCNA guideline on the management of blood cholesterol: a report of the American College of Cardiology/ American Heart Association task force on clinical practice guidelines. Circulation. 2019;139:e1082-143.

6. Smolen JS et al. EULAR recommendations for the management of rheumatoid arthritis with synthetic and biological disease-modifying antirheumatic drugs: 2013 update. Ann Rheum Dis. 2014;73(3):492-509.

7. Khanna D et al. 2012 American College of Rheumatology guidelines for management of gout. Part 1 : systematic nonpharmacologic and pharmacologic therapeutic approaches to hyperuricemia. Arthritis Care Res. 2012;64(10):143146.

8. Van Vollenhoven RF et al. Treatto-target in systemic lupus erythematosus: recommendations from an international task force. Ann
Rheum Dis. 2014;73(6):958-67.

9. Grigor $\mathrm{C}$ et al. Effect of a treatment strategy of tight control for rheumatoid arthritis (the TICORA study): a single-blind randomised controlled trial. Lancet. 2004;364(9430):263-9

10. Perez-Ruiz F et al. Effect of uratelowering therapy on the velocity of size reduction of tophi in chronic gout. Arthritis Care Res. 2002;47(4):356-60.

11. Smolen JS et al. Treating axial spondyloarthritis and peripheral spondyloarthritis, especially psoriatic arthritis, to target: 2017 update of recommendations by an international task force. 2017;77(1):3-17.

12. Gossec $L$ et al. EULAR recommendations for the management of psoriatic arthritis with pharmacological therapies: 2019 update. Ann Rheum Dis. 2020;79(6):S700-12. 
13. Singh JA et al. Special article: 2018 American College of Rheumatology/ National Psoriasis Foundation guideline for the treatment of psoriatic arthritis. Arthritis Rheumatol. 2019;71:5-32.

14. Van Der Heijde D et al. 2016 update of the ASAS-EULAR management recommendations for axial spondyloarthritis. Ann Rheum Dis. 2017;76(6):978-91.

15. Ward MM et al. American College of Rheumatology/ Spondylitis Association of America/Spondyloarthritis Research and Treatment Network 2015 recommendations for the treatment of ankylosing spondylitis and nonradiographic axial spondyloarthritis. Arthritis Rheumatol. 2016;68(2):282-98.

16. Machado PM, Deodhar A. Treat-totarget in axial spondyloarthritis: gold standard or fools' gold? Curr Opin Rheumatol. 2019;31(4):344-8.

17. Ramiro $\mathrm{S}$ et al. Higher disease activity leads to more structural damage in the spine in ankylosing spondylitis: 12-year longitudinal data from the OASIS cohort. Ann Rheum Dis. 2014;73:1455-61

18. Poddubnyy $D$ et al. High disease activity according to the ankylosing spondylitis disease activity score is associated with accelerated radiographic spinal progression in patients with early axial spondyloarthritis: results from the German Spondyloarthritis Inception cohort. Ann Rheum Dis. 2016;75(12):2114-8.

19. Molnar $\mathrm{C}$ et al. TNF blockers inhibit spinal radiographic progression in ankylosing spondylitis by reducing disease activity: results from the Swiss Clinical Quality Management cohort. Ann Rheum Dis. 2018;77(1):63-9.

20. Van Der Heijde D et al. The effect of golimumab therapy on disease activity and health-related quality of life in patients with ankylosing spondylitis: 2-year results of the GO-RAISE trial. J Rheumatol. 2014:41(6):1095-103.

21. Van der Heijde D et al. ASAS4O and ASDAS clinical responses in the ABILITY-1 clinical trial translate to meaningful improvements in physical function, health-related quality of life and work productivity in patients with non-radiographic axial spondyloarthritis. Rheumatology (Oxford). 2016;55(1):80-8.

22. Coates LC et al. Group for research and assessment of psoriasis and psoriatic arthritis 2015 treatment recommendations for psoriatic arthritis. Arthritis Rheumatol. 2016;68(5):1060-71.

23. Coates LC et al. Group for research and assessment of psoriasis and psoriatic arthritis/outcome measures in rheumatology consensus-based recommendations and research agenda for use of composite measures and treatment targets in psoriatic arthritis. Arthritis Rheumatol. 2018;70:345-55.

24. Schoels MM et al. Treating axial and peripheral spondyloarthritis, including psoriatic arthritis, to target: results of a systematic literature search to support an international treat-to-target recommendation in spondyloarthritis. Ann Rheum Dis. 2014;73(1):238-42.

25. Hagège $B$ et al. Remission and low disease activity in psoriatic arthritis publications: a systematic literature review with metaanalysis. Rheumatology (Oxford). 2020;59(8):1818-25.

26. Coates LC et al. Comparison of remission and low disease activity states with DAPSA, MDA and VLDA in a clinical trial setting in psoriatic arthritis patients: 2-year results from the FUTURE 2 study. Semin Arthritis Rheum. 2020;50(4):709-18.

27. Eberl G et al. Development of a disease activity index for the assessment of reactive arthritis (DAREA). Rheumatology. 2000;39(2):148-55.

28. Schoels $M$ et al. Application of the DAREA/DAPSA score for assessment of disease activity in psoriatic arthritis. Ann Rheum Dis. 2010;69(8):1441-7.

29. Husic R et al. Disparity between ultrasound and clinical findings in psoriatic arthritis. Ann Rheum Dis. 2014;73:1529-36.

30. Schoels MM et al. Disease activity in psoriatic arthritis (PsA): defining remission and treatment success using the DAPSA score. Ann Rheum Dis. 2016;75:811-8.

31. Coates LC, Helliwell PS. Treat to target in psoriatic arthritis-evidence, target, research agenda. Curr Rheumatol Rep. 2015;17(6):15-20.

32. Coates LC et al. Effect of tight contro of inflammation in early psoriatic arthritis (TICOPA): a UK multicentre, open-label, randomised controlled trial. Lancet. 2015;386(10012):248998.

33. Mease P et al. Comparison of disease control thresholds in psoriatic arthritis: results from the Corrona psoriatic arthritis / spondyloarthritis registry. Abstract 0884 . ACR Convergence, 7 November, 2020.

34. McInnes IB et al. Secukinumab versus adalimumab for treatment of active psoriatic arthritis (EXCEED): a double-blind, parallel-group, randomised, activecontrolled, phase 3b trial. Lancet. 2020;395(10235):1496-505. Erratum in: Lancet. 2020;395(10238):1694.

35. Mease PJ et al. A head-to-head comparison of the efficacy and safety of ixekizumab and adalimumab in biological-naïve patients with active psoriatic arthritis: 24-week results of a randomised, open-label, blindedassessor trial. Ann Rheum Dis. 2020;79(1):123-31.

36. Smolen JS et al. Target outcomes in psoriatic arthritis: simultaneous achievement of ACR50-Psoriasis Area And Severity Index 100 and beyond: insights from open-label, assessorblinded study at week 24. Abstract 0319. ACR Convergence, 6 November, 2020.

37. Pittam B et al. Prevalence of extraarticular manifestations in psoriatic arthritis: A systematic review and meta-analysis. Rheumatology (Oxford). 2020;59(9):2199-206.

38. Charlton R et al. Risk of uveitis and inflammatory bowel disease in people with psoriatic arthritis: a populationbased cohort study. Ann Rheum Dis 2018;77(2):277-80

39. Deodhar A et al. Guselkumab in patients with active psoriatic arthritis who were biologic-naive or had previously received TNFa inhibitor treatment (DISCOVER-1): a double-blind, randomised, placebocontrolled phase 3 trial. Lancet. 2020;395(10230):1115-25.

40. Bravo A, Kavanaugh A. Bedside to bench: defining the immunopathogenesis of psoriatic arthritis. Nat Rev Rheumatol. 2019;15(11):645-56

41. Fragoulis GE, Siebert S. Treatment strategies in axial spondyloarthritis: What, when and how? Revmatol. 2020;59(Suppl 4):IV79-89.

42. Coates $L$ et al. Does treat to target or achieving remission improve radiographic outcome in $\mathrm{PSA}$ ? Arthritis Rheumatol. 2016:68:2118-21.

43. O'Dwyer JL et al. Cost-effectiveness of tight control of inflammation in early psoriatic arthritis: economic analysis of a multicenter randomized controlled trial. Arthritis Care Res (Hoboken). 2018;70:462-8.

44. Beckers $E$ et al. Is treat-to-target applied in axial spondyloarthritis in daily Practice? An observational study in three rheumatology centers with an electronic monitoring tool available. 2019;20:2019.

45. Dures $E$ et al. Mixed methods study of clinicians' perspectives on barriers to implementation of treat to target in psoriatic arthritis. Ann Rheum Dis 2020;79(8):1031-6

46. Garrett S et al. A new approach to defining disease status in ankylosing spondylitis: the Bath Ankylosing Spondylitis Disease Activity Index. J Rheumatol. 1994;21(12):2286-91.

47. Machado P et al. Ankylosing spondylitis disease activity score (ASDAS): defining cut-off values for disease activity states and improvement scores. Ann Rheum Dis. 
2011;70:47-53.

48. Machado PM et al. Ankylosing spondylitis disease activity score (ASDAS): 2018 update of the nomenclature for disease activity states. Ann Rheum Dis. 2018;77:153940.

49. Lukas C et al. Development of an ASAS-endorsed disease activity score (ASDAS) in patients with ankylosing spondylitis. Ann Rheum Dis. 2009;68(1):18-24.

50. Anderson JJ et al. Ankylosing spondylitis assessment group preliminary definition of shortterm improvement in ankylosing spondylitis. Arthritis Rheum. 2001;44(8):1876-86.

51. Kiltz $U$ et al. Measurement properties of the ASAS health index: results of a global study in patients with axial and peripheral spondyloarthritis. Ann Rheum Dis. 2018;77(9):1311-7.

52. Liew JW, Dubreuil M. Treat to target in axial spondyloarthritis: pros, cons, and future directions. Rheum Dis Clin North Am. 2020;46:343-56.

53. Spoorenberg $A$ et al. Measuring disease activity in ankylosing spondylitis: patient and physician have different perspectives. Rheumatology. 2005;44:789-95.

54. Machado P, Van Der Heijde D. How to measure disease activity in axial spondyloarthritis? Curr Opin Rheumatol. 2011;23(4):339-45.

55. Moltó A et al. Thu0370 clusterrandomized pragmatic clinical trial evaluating the potential benefit of a tight-control and treat-to-target strategy in axial spondyloarthritis: the results of the TICOSPA trial. Ann Rheum Dis. Abstract 1444. ACR Convergence, 8 November, 2020.

56. Stolwijk $\mathrm{C}$ et al. Prevalence of extraarticular manifestations in patients with ankylosing spondylitis: a systematic review and meta-analysis. Ann Rheum Dis. 2015;74(1):65-73.

57. Haroon $\mathrm{N}$ et al. The impact of tumor necrosis factor a inhibitors on radiographic progression in ankylosing spondylitis. Arthritis Rheum. 2013;65(10):2645-54

58. Baraliakos $X$ et al. Continuous longterm anti-TNF therapy does not lead to an increase in the rate of new bone formation over 8 years in patients with ankylosing spondylitis. Ann Rheum Dis. 2014;73(4):710-5.

59. Deodhar A et al. Tofacitinib for the treatment of ankylosing spondylitis: a phase III, randomised, doublecontrolled study. 2021;DOI:10.1136/ annrheumdis-2020-219601.

60. AbbVie. A Study Treating Participants With Early Axial Spondyloarthritis (axSpA) Taking an Intense Treatment Approach Versus Routine Treatment (STRIKE). NCTO2897115. https:// clinicaltrials.gov/ct2/show/ NCTO2897115.

61. Ward MM et al. 2019 Update of the American College of Rheumatology/ Spondylitis Association of America/ Spondyloarthritis Research and Treatment Network recommendations for the treatment of ankylosing spondylitis and nonradiographic axial spondyloarthritis. Arthritis Rheumatol. 2019;71(10):1599-613.

62. Haraoui B et al. Treating rheumatoid arthritis to target: multinational recommendations assessment questionnaire. Ann Rheum Dis. 2011;70(11):1999-2002.

63. Gvozdenović E et al. When rheumatologists report that they agree with a guideline, does this mean that they practise the guideline in clinical practice? Results of the international recommendation implementation study (IRIS). RMD Open. 2016;2(1):e000221.

64. Vermeer $M$ et al. Adherence to a treat-to-target strategy in early rheumatoid arthritis: results of the DREAM remission induction cohort. Arthritis Res Ther. 2012;14(6):R254.

65. New J et al. Specialist nurse-led intervention to treat and control hypertension and hyperlipidemia in diabetes (SPLINT): a randomized controlled trial. Diabetes Care. 2003;26(8):2250-5.

66. Clark CE et al. Nurse led interventions to improve control of blood pressure in people with hypertension: systematic review and meta-analysis. BMJ. 2010;341:c3995.

67. Newman E et al. Treat to target opportunities - design, testing, and adoption of a novel EHR-integrated electronic system to engage rheumatologists and capture decision making. Arthritis Rheumatol. Abstract 1862. ACR/ARP Annual Meeting, 11 November, 2019. 